\title{
Optimization of Anterior Incision Placement for Distal Biceps Repair
}

\author{
Nikolai Klebanov ${ }^{1}$, David H. Wei ${ }^{2}$, Brendan J. Harrison ${ }^{3}$, Hervey L. Kimball ${ }^{4}$ \\ 1. Dermatology, Massachusetts General Hospital, Boston, USA 2. Orthopaedic Surgery, Greenwich Hospital, \\ Greenwich, USA 3. Internal Medicine, Steward Carney Hospital/Tufts University School of Medicine, Dorchester, USA 4. \\ Orthopedic Surgery, New England Baptist Hospital, Boston, USA
}

Corresponding author: Nikolai Klebanov, nikolai.klebanov@gmail.com

\section{Abstract}

\section{Introduction}

Damage to the posterior interosseous nerve (PIN) is a known complication when using a cortical button during distal biceps tendon repair. Prior studies show that the trajectory of the drill through the biceps tuberosity can affect the distance from the PIN. We develop a mathematical model to predict the location of the tuberosity based on a palpable bony landmark and patient demographic factors.

\section{Methods}

The medical charts and elbow radiographs of $(n=82)$ adult patients were retrospectively reviewed. Using standard radiographic software, two observers measured the distance from the olecranon tip to the center of the biceps tuberosity. Multivariate regression analysis was used to build a linear model. The model was cross-validated with five arms from three distinct cadavers. A surgical wire was guided into the volar aspect of each forearm using the model, and a dissection was then performed to assess the proximity of the surgical wire to the insertion of the biceps tendon on the radial tuberosity.

\section{Results}

Olecranon-tuberosity distance (OTD) ranged from $52.3 \mathrm{~mm}$ to $77.2 \mathrm{~mm}$ (mean $66.5 \mathrm{~mm}$ ). Univariate analyses revealed males had significantly longer OTD (mean $69.3 \mathrm{~mm}$ ) compared to females (mean $61.2 \mathrm{~mm}$, t-test, $\mathrm{p}$ $<0.001)$. Increased body mass index (BMI) weakly correlated with increased distance (Pearson's $\mathrm{r}=0.22, \mathrm{p}=$ $0.048)$. Height showed strong positive correlation with increased distance $(r=0.77, p<0.001)$. Multivariate regression revealed that significant predictive factors for olecranon-tuberosity distance were height (coefficient $=35.8, \mathrm{p}<0.001)$, BMI (coefficient $=0.14, \mathrm{p}=0.032$ ), and male $\operatorname{sex}($ coefficient $=3.17, \mathrm{p}=$ 0.0039). The average error in the cadaveric validation, measured as distance from the surgical wire to the distal biceps insertion was $1.8 \mathrm{~mm}$.

Received 08/06/2018 Review began 08/08/2018 Review ended 08/13/2018 Published 08/14/2018

\section{() Copyright 2018}

Klebanov et al. This is an open access article distributed under the terms of the Creative Commons Attribution License CC-BY 3.0., which permits unrestricted use, distribution, and reproduction in any medium, provided the original author and source are credited.

\section{Conclusion}

A highly accurate mathematical model can be used to predict the location of the biceps tuberosity in relation to the palpable tip of the olecranon, based only on height, BMI, and sex of the patient. Knowledge of this distance can guide accurate placement of the skin incision when a transverse single-incision approach is utilized for repair of the distal biceps tendon using a cortical button. Diagnostics showed the model to be less accurate near the extremes of the measurement. Since patients with a target incision point far removed from average would most benefit from such a model, we will continue by identifying and enrolling patients at the low and high ends of the range. We further hypothesize that the technique described above could be similarly applied to benefit other procedures.

Categories: Medical Simulation, Radiology, Orthopedics

Keywords: biceps tendon, biceps tendon rupture, radiographic anatomy

\section{Introduction}

Distal biceps tendon rupture occurs commonly in adult men [1], resulting from sudden eccentric contraction of the biceps brachii muscle [2]. Operative repair is the preferred treatment of distal biceps tendon rupture $[3,4]$. Anatomical reinsertion with a one- or two-incision technique yields superior outcomes among multiple surgical techniques [5-7], with single-incision techniques rising in popularity in an effort to reduce post-operative complications $[8,9]$. For instance, the cortical button is a commonly-used technique that provides robust strength with a single anterior incision [10-12].

The cortical button repair strategy carries a risk of posterior interosseous nerve (PIN) injury, which occurs with an estimated frequency of $3 \%[13,14]$. This repair is performed by drilling a tunnel through the radius, starting at the radial tuberosity and exiting dorsally. The metal cortical button is sutured to the distal 


\section{Cureus}

portion of the biceps brachii and pulled through the tunnel. The button is then flipped on the dorsal aspect of the radius to affix the tendon on the bone. Theoretically, the PIN could be damaged by entrapment with the implant on the dorsal cortex of the proximal radius [15]. This damage may result in transient PIN palsy, and is an uncommon, but severe complication of distal biceps tendon rupture repair [16, 17].

Precise drill orientation through the radial tuberosity is important in avoiding PIN injury [13]. In order to fully visualize the radial tuberosity and achieve optimal drill angle and position, it is helpful for the surgeon to place the initial surgical incision as close as possible to the center of the tuberosity. Thus, the aim of this study was to create a set of equations or rules to guide the surgeon in making the optimal anterior incision for the procedure. Optimizing incision placement could then possibly make it easier to correctly orient the drill and to avoid PIN injury. We hypothesized that the olecranon tip, a palpable bony landmark, could be used as a reference point to estimate distance to the radial tuberosity center. We aimed to investigate how the distance from the olecranon tip to the radial tuberosity could be reliably estimated from patient demographic information.

\section{Materials And Methods \\ Patient selection}

All elbow X-rays dating between 2013 and 2015 at a single institution were reviewed. Patients were included in the study if at least two views (anteroposterior and lateral) were present. Patients were excluded based on the following criteria: a) prior surgery for distal biceps tendon repair; b) prior extensive surgery introducing any modifications or implants, including but not limited to full elbow arthroplasty; c) evidence of any fracture or bony deformity, malunion, or nonunion; d) evidence of severe degenerative change or osteoarthritis of the elbow joint. The 82 patients with plain film X-rays dated between 2013 and 2015 who satisfied the above criteria consisted of 54 men and 28 women with age ranging from 16 to 84 years (mean 48.2 years). Each of the 82 plain film X-ray series was confirmed to be from a distinct patient.

\section{Radiographic analysis}

Proximal and distal borders of the radial tuberosity were identified, and the center of the tuberosity was marked at the center of this length. Using anteroposterior (AP) views, the distance between the most proximal tip of the olecranon process and the center of the radial tuberosity (D) was measured (Figure 1).

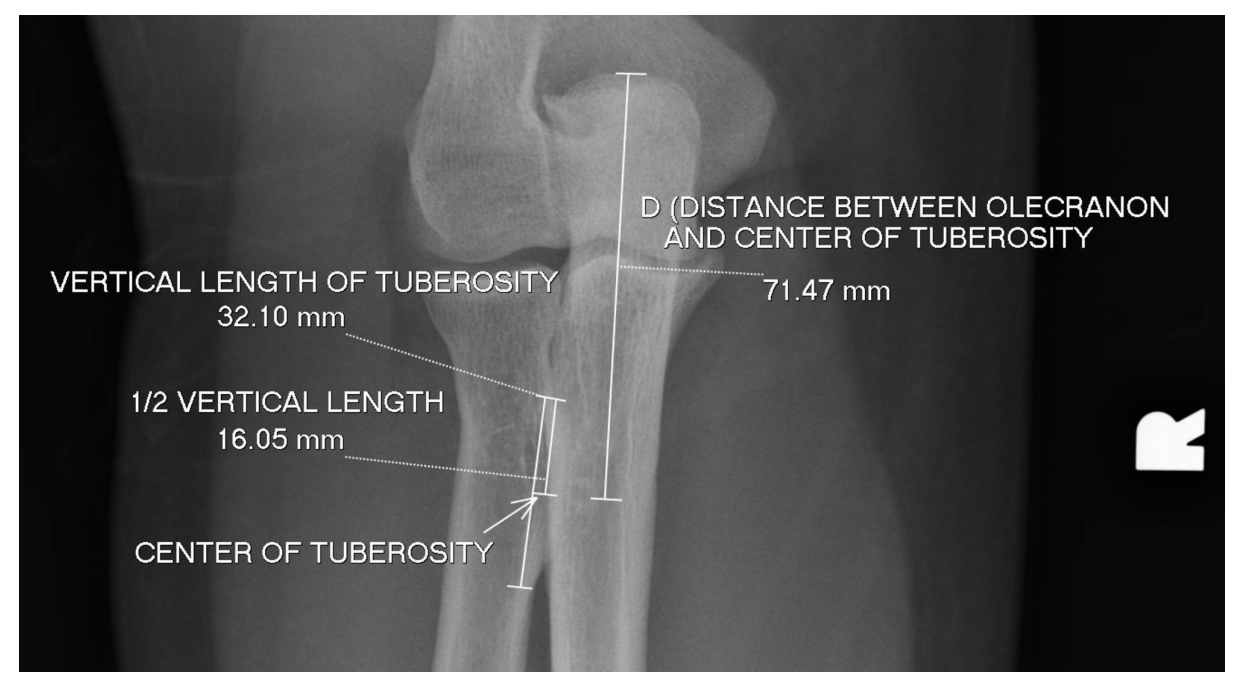

\section{FIGURE 1: Measurement of olecranon to tuberosity distance.}

Radiographic measurement of the distance in $\mathrm{mm}$ between the olecranon tip and the center of the radial tuberosity using anteroposterior (AP) plain film X-rays.

If this was possible on more than one AP view, the mean of these measurements was recorded. Multivariate linear regression was used to relate this distance to age, sex, height, and body mass index (BMI).

\section{Cadaveric study}

Five cadaveric arms that had been harvested from three patients (Table 1) at mid-humerus and fully intact distally were obtained. 


\section{Cureus}

\begin{tabular}{|c|c|c|c|c|c|c|c|c|}
\hline Cadaver & Age & Height (m) & Weight (kg) & BMI & Sex & Arm & Predicted distance $(\mathrm{mm})$ & Wire placement error (mm) \\
\hline 1 & 29 & 1.60 & 50.0 & 19.5 & $F$ & R & 60.2 & 2 \\
\hline 1 & 29 & 1.60 & 50.0 & 19.5 & $F$ & $\mathrm{~L}$ & 60.2 & 5 \\
\hline 2 & 68 & 1.65 & 63.6 & 23.4 & $\mathrm{M}$ & R & 65.7 & 2 \\
\hline 2 & 68 & 1.65 & 63.6 & 23.4 & M & $\mathrm{L}$ & 65.7 & 0 \\
\hline 3 & 79 & 1.78 & 81.7 & 25.8 & $\mathrm{M}$ & $\mathrm{L}$ & 70.7 & 2 \\
\hline
\end{tabular}

\section{TABLE 1: Cadavers' clinical characteristics and surgical wire model validation.}

Five cadaveric arms from three patients were used to validate the statistical model predicted by radiographical measurements. The predicted distance ranged from $60.2 \mathrm{~mm}$ to $70.7 \mathrm{~mm}$. The mean error (distance between surgical wire and center of tuberosity) was $1.8 \mathrm{~mm}$.

BMI: Body mass index.

Donors' sex, height, and BMI were used to calculate the expected olecranon tip to biceps tuberosity center length using the multivariate model equations. This distance was marked along the posterior aspect of the elbow held at $90^{\circ}$ flexion and maximum supination. A $16 \mathrm{~mm}$-diameter surgical wire was driven vertically through the proximal radius with the forearm in maximum supination at the marked site. A transverse volar incision was made in each specimen, centered over the guide pin at the location marked previously. Dissection was carried down toward the radial tuberosity. The distal tendon of the biceps was identified on each specimen, and its insertion onto the radial tuberosity was visualized. The error between the surgical wire and the center of tendon insertion was measured.

\section{Results}

The distance between the olecranon tip and the center of the radial tuberosity ranged from $52.3 \mathrm{~mm}$ to 77.2 $\mathrm{mm}$ (mean $66.5 \mathrm{~mm}$ ) (Figure $2 \mathrm{~A}$ ). Males had significantly longer olecranon-tuberosity distance at a mean $69.3 \mathrm{~mm}$ compared to females, who had mean distance of $61.2 \mathrm{~mm}$ ( $\mathrm{t}$-test, $\mathrm{p}=5.9 \times 10^{-10}$ ) (Figure $2 B$ ). Increased BMI weakly correlated with increased distance (Pearson's $r=0.22, p=0.048$ ). Height showed strong positive correlation with increased distance $(r=0.77, \mathrm{p}<0.001)$ (Figure $2 C, 2 D)$.
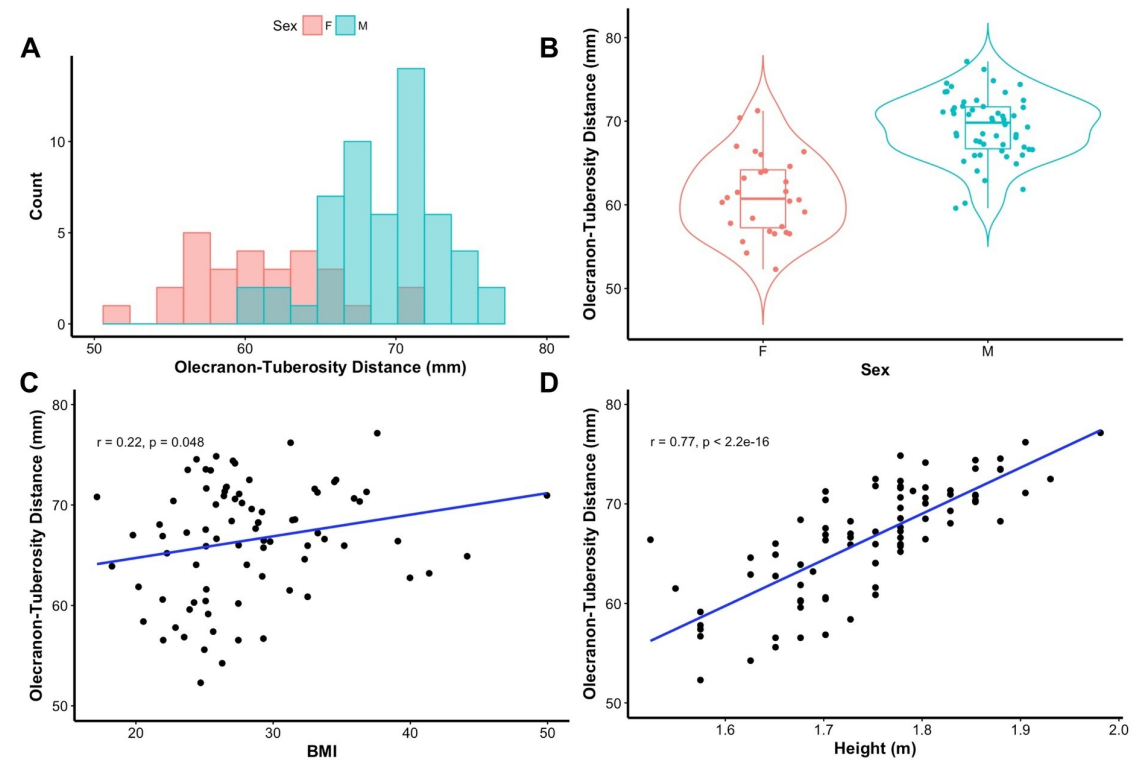

FIGURE 2: Association between clinical factors and olecranontuberosity distance.

A) Histogram of distance measured radiographically between the olecranon tip and the center of the radial tuberosity. B) Sex was significantly associated with olecranon-tuberosity distance by univariate t-test ( $p<$ 0.001 ), with males having an average length of $69 \mathrm{~mm}$, and females $61 \mathrm{~mm}$. C) Body mass index (BMI) significantly correlated with distance $(p<0.05)$. D) Height significantly correlated with distance $(p<0.001)$. 
Multivariate regression revealed that significant predictive factors for olecranon-tuberosity distance were height $($ coefficient $=35.8, \mathrm{p}<0.001$ ), BMI (coefficient $=0.14, \mathrm{p}=0.032$ ), and male sex (coefficient $=3.17, \mathrm{p}=$ 0.0039 ). The overall significance of the model was $p<0.001$, with R2 value of 0.6685 , suggesting that $67 \%$ of variance is accounted for by the model.

Using the coefficients of the multivariate regression model, we developed an algebraic formula to predict the olecranon-tuberosity distance (OTD):

$O T D=35.9 *($ Height $)+0.14 *(B M I)+3.17$

$*$ Male $_{\text {yes }=1}$

By model predictions, we estimated the approximate tuberosity location relative to the olecranon tip in the cadaveric arms. The error (in $\mathrm{mm}$ ) between the surgical wire placement and the center of the tuberosity in the cadaveric study ranged from $0 \mathrm{~mm}$ to $5 \mathrm{~mm}$ (mean $1.8 \mathrm{~mm}$ ) (Table 1 ).

\section{Discussion}

While prior studies have investigated the distance from the biceps tendon insertion on the radial tuberosity [18-21] and to estimate the location of the PIN [22, 23], this is the first study to our knowledge using the olecranon tip as a landmark. The results of our univariate and multivariate analyses suggest that distance from the olecranon tip of the elbow to the center of the bicipital tuberosity (OTD) significantly depends on height, BMI, and sex. The positive correlations of OTD to height and BMI are logical given anatomical proportionality of different individuals. Furthermore, our finding that males had a longer olecranontuberosity distance of $69.3 \mathrm{~mm}$ compared to females at $61.2 \mathrm{~mm}$ is consistent with prior studies. For instance, men were found to have a longer distance between the radial head and the biceps tuberosity (20.9 $\mathrm{mm}$ ) compared to women $(17.7 \mathrm{~mm})$ [21]. Cadaveric study suggested that the model performed with $2 \mathrm{~mm}$ accuracy.

The PIN is at risk of damage during a cortical button repair, as the repair is performed blindly without a second incision dorsally to confirm no nerve entrapment. We have used radiographic modeling of elbow anatomy in the hopes of guiding surgical incision placement. Using the multivariate regression model, we propose two tables, for male and female patients, as easily-accessible guides for orthopedic surgeons (Figure 3).

\section{DISTANCE BETWEEN OLECRANON TIP TO OPTIMAL INCISION}

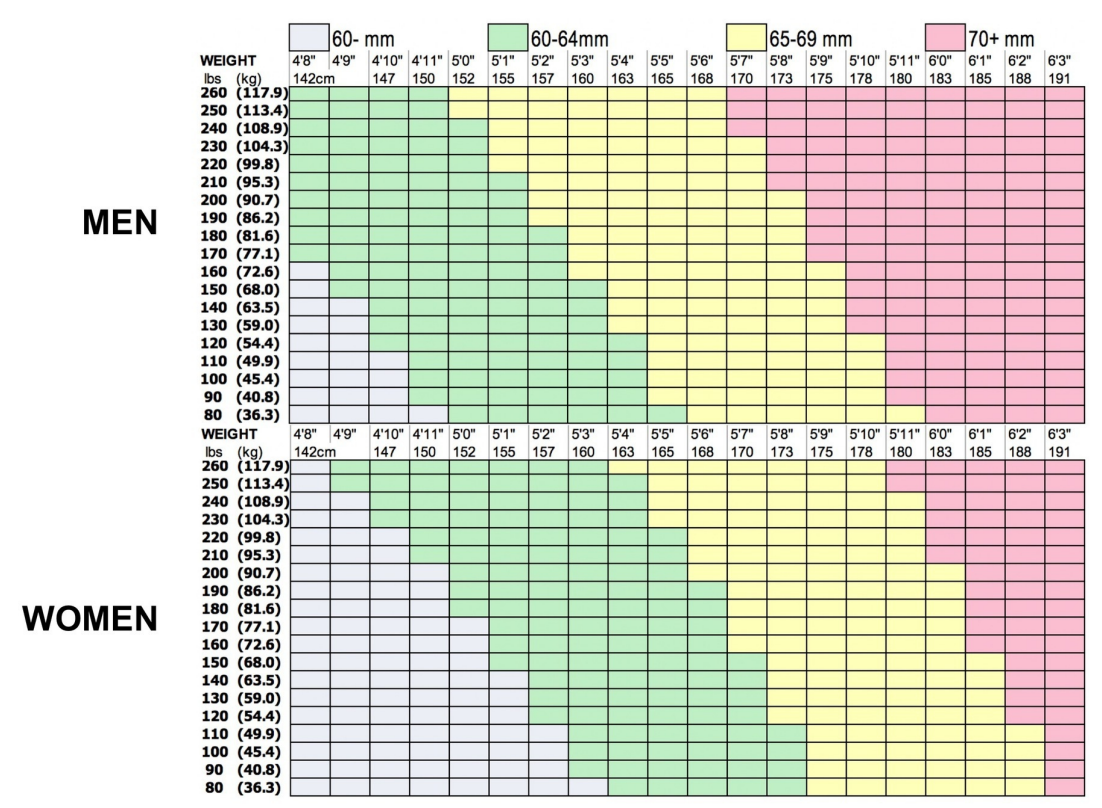

FIGURE 3: Optimal biceps tendon surgical incision placement rules.

Guides for estimation of optimal volar incision placement based on sex, height, and weight.

This study had several limitations. First, the cadaveric study focused on a small number of samples, and thus 
there were few data points for model validation. Second, the baseline variation between samples was within $20 \mathrm{~mm}$, which is a narrow range. During surgery, a dissection is usually performed first to fully visualize the area, and thus the biceps tuberosity is likely to be visualized in its entirety even if the initial incision was optimized. Finally, this study did not prospectively evaluate whether implementation of this technique changes the rate of PIN injury. Given that the already low rate of PIN injury of approximately 3\%, it is possible that minor adjustments to incision placement using the proposed technique may not significantly decrease PIN injury risk. Future prospective studies comparing PIN injury in cortical button repairs with and without incision placement optimization may be useful in validating the proposed optimization effort.

\section{Conclusions}

PIN damage is a known complication of single-incision biceps tendon rupture repair with the cortical button technique. This study investigated the use of patient demographic factors to predict the distance between the olecranon tip of the elbow and the center of the radial tuberosity. Thus, using such a model, the olecranon tip can be used as a surgical landmark to estimate the preferred location for incision placement. The model was validated with surgical wire placement in five cadaveric arms and achieved accuracy within 2 $\mathrm{mm}$. We propose that a radiographic modeling technique could be similarly applied to the study of other orthopedic procedures.

\section{Additional Information \\ Disclosures}

Human subjects: Consent was obtained by all participants in this study. New England Baptist Hospital issued approval 694182. This submission ([694182-1] Locating the optimal transverse incision for repair of the distal biceps tendon) has been reviewed and approved by the NEBH IRB, Assurance \# FWA 00009165. Animal subjects: All authors have confirmed that this study did not involve animal subjects or tissue. Conflicts of interest: In compliance with the ICMJE uniform disclosure form, all authors declare the following: Payment/services info: All authors have declared that no financial support was received from any organization for the submitted work. Financial relationships: All authors have declared that they have no financial relationships at present or within the previous three years with any organizations that might have an interest in the submitted work. Other relationships: All authors have declared that there are no other relationships or activities that could appear to have influenced the submitted work.

\section{References}

1. Keener JD: Controversies in the surgical treatment of distal biceps tendon ruptures: single versus doubleincision repairs. J Shoulder Elbow Surg. 2011, 20:113-125. 10.1016/j.jse.2010.11.009

2. Leighton MM, Bush-Joseph CA, Bach BR Jr: Distal biceps brachii repair. Results in dominant and nondominant extremities. Clin Orthop Relat Res. 1995, 317:114-121.

3. Chillemi C, Marinelli M, De Cupis V: Rupture of the distal biceps brachii tendon: conservative treatment versus anatomic reinsertion--clinical and radiological evaluation after 2 years. Arch Orthop Trauma Surg. 2007, 127:705-708. 10.1007/s00402-007-0326-7

4. Baker BE, Bierwagen D: Rupture of the distal tendon of the biceps brachii. Operative versus non-operative treatment. J Bone Joint Surg Am. 1985, 67:414-417.

5. Miyamoto RG, Elser F, Millett PJ: Distal biceps tendon injuries. J Bone Joint Surg Am. 2010, 92:2128-2138. 10.2106/JBJS.I.01213

6. Sutton KM, Dodds SD, Ahmad CS, Sethi PM: Surgical treatment of distal biceps rupture. J Am Acad Orthop Surg. 2010, 18:139-148.

7. Moosmayer S, Odinsson A, Holm I: Distal biceps tendon rupture operated on with the Boyd-Anderson technique: follow-up of 9 patients with isokinetic examination after 1 year. Acta Orthop Scand. 2000, 71:399-402. 10.1080/000164700317393411

8. Boyd HB, Anderson LD: A method for reinsertion of the distal biceps brachii tendon . J Bone Joint Surg. 1961, 43:1041-1043. 10.2106/00004623-196143070-00012

9. Greenberg JA, Fernandez JJ, Wang T, Turner C: EndoButton-assisted repair of distal biceps tendon ruptures . J Shoulder Elbow Surg. 2003, 12:484-490. 10.1016/S1058-2746(03)00173-3

10. Chavan PR, Duquin TR, Bisson LJ: Repair of the ruptured distal biceps tendon: a systematic review . Am J Sports Med. 2008, 36:1618-1624. 10.1177/0363546508321482

11. Kettler M, Lunger J, Kuhn V, Mutschler W, Tingart MJ: Failure strengths in distal biceps tendon repair. Am J Sports Med. 2007, 35:1544-1548. 10.1177/0363546507300690

12. Mazzocca AD, Burton KJ, Romeo AA, Santangelo S, Adams DA, Arciero RA: Biomechanical evaluation of 4 techniques of distal biceps brachii tendon repair. Am J Sports Med. 2007, 35:252-258. 10.1177/0363546506294854

13. Nigro PT, Cain R, Mighell MA: Prognosis for recovery of posterior interosseous nerve palsy after distal biceps repair. J Shoulder Elbow Surg. 2013, 22:70-73. 10.1016/j.jse.2012.08.001

14. Panagopoulos A, Tatani I, Tsoumpos P, Ntourantonis D, Pantazis K, Triantafyllopoulos IK: Clinical outcomes and complications of cortical button distal biceps repair: a systematic review of the literature. J Sports Med. 2016, 2016:11. 10.1155/2016/3498403

15. Lo EY, Li CS, Van den Bogaerde JM: The effect of drill trajectory on proximity to the posterior interosseous nerve during cortical button distal biceps repair. Arthroscopy. 2011, 27:1048-1054.

10.1016/j.arthro.2011.03.084

16. Cain RA, Nydick JA, Stein MI, Williams BD, Polikandriotis JA, Hess AV: Complications following distal biceps 


\section{Cureus}

repair. J Hand Surg Am. 2012, $37: 2112-2117$. 10.1016/j.jhsa.2012.06.022

17. Stearns KL, Sarris I, Sotereanos DG: Permanent posterior interosseous nerve palsy following a two-incision distal biceps tendon repair. Orthopedics. 2004, 27:867-868.

18. Hutchinson HL, Gloystein D, Gillespie M: Distal biceps tendon insertion: an anatomic study . J Shoulder Elbow Surg. 2008, 17:342-346.10.1016/j.jse.2007.05.005

19. Mazzocca AD, Cohen M, Berkson E, Nicholson G, Carofino BC, Arciero R, Romeo AA: The anatomy of the bicipital tuberosity and distal biceps tendon. J Shoulder Elbow Surg. 2007, 16:122-127. 10.1016/j.jse.2006.04.012

20. Athwal GS, Steinmann SP, Rispoli DM: The distal biceps tendon: footprint and relevant clinical anatomy . J Hand Surg Am. 2007, 32:1225-1229. 10.1016/j.jhsa.2007.05.027

21. Cho CH, Song KS, Choi IJ, Kim DK, Lee JH, Kim HT, Moon YS: Insertional anatomy and clinical relevance of the distal biceps tendon. Knee Surg Sports Traumatol Arthrosc. 2011, 19:1930-1935. 10.1007/s00167-0111586-x

22. Diliberti T, Botte MJ, Abrams RA: Anatomical considerations regarding the posterior interosseous nerve during posterolateral approaches to the proximal part of the radius. J Bone Joint Surg Am. 2000, 82:809-813. 10.2106/00004623-200006000-00007

23. Tubbs RS, Salter EG, Wellons JC 3rd, Blount JP, Oakes WJ: Superficial surgical landmarks for identifying the posterior interosseous nerve. J Neurosurg. 2006, 104:796-799. 10.3171/jns.2006.104.5.796 